\title{
Study on Bidirectional-Charger for Electric Vehicle Applied to Power Dispatching in Smart Grid
}

\author{
Topics: Renewable Energy and Smart Grid Technologies
}

\begin{abstract}
It will be one of the most important content to take the electric vehicle batteries as distributed energy storage units which can accept the power dispatching, and now bidirectional charger is the key power electronic device that has been used to connect power grid to the electric vehicle battery. In this paper, a two-stage bidirectional power converter made up of T-type neutral point clamped (NPC) three-phase three-level (TL) AC/DC and dual active bridge (DAB) DC/DC is studied, And the direct power control strategy is adopted to accept power dispatching from power grid. With high reliability, efficiency and high power quality as the goal, a novel space vector modulation (SVPWM) is proposed to avoid the terminal voltage distortion of bridge legs, and the series-parallel-connection DAB converter is presented to further improve efficiency. Finally, experimental results verify the feasibility of circuit topology and control strategy.
\end{abstract}

\section{INTRODUCTION}

With the development of application of electric vehicles (EVs) and smart grid, the EVs, as mobile storage terminal as shown in Fig.1, can absorb active power energy from grid to be charged by some certain battery charger, on the other hand generate power from vehicle to grid (V2G) to be discharged by the same battery charger as well[1]. More and more attention has been paid on V2G technology and V2G techniques and its reactive power operation has become one of the most important application in future smart grid[2]. Of course, it is the bidirectional battery charger that plays an key role on bidirectional power flow between grid and electric vehicle.

Many of battery chargers are only with basic function of charging electricity and the most of charging infrastructure are without consideration of bidirectional power flow, let alone the function of reactive power operation [3]. The document [4] related a kind of Buck/Boost circuit which can both charge and discharge storage battery, however, the referred Buck/Boost circuit in [4] is not isolated in electrical connection, as a consequence, reducing safety and some certain reliability [5].

For the isolation purpose, the circuits cascaded by bidirectional AC/DC and DAB-based DC/DC have been studied in document [6,7], and serve as main circuit of battery charger to improve the safety of the system. One of merits of this kind of circuit topology is that the turn ratio of high-frequency transformer can be configured to match the input and output voltages, which are not limited to effective duty cycle again. However, efficiency will be frustrated if turn ratio is much larger. It is because the turn-on resistance or voltage drop of high-voltage-rated switch is much higher than that of low-voltage- rated switch on the high-voltage side, and the current stress of switch is much higher on the low-voltage side when operating in larger power level. For this kind of situation, series-parallel DAB (SPDAB) converter is put forward in this paper to improve efficiency and power density.

As to the front-end AC/DC converter in bidirectional charger shown in the Fig.1, many circuits, if only they can operate in four quadrants, can be employed. Here, T-type NPC three-phase TL AC/DC converter serves as the front-end circuit. In order to suppress electromagnetic interference (EMI), many modulation methods have been presented by researchers [8]. Among these, a improved space vector modulation (ISVPWM) is proposed to effectively decrease common voltage, but ISVPWM is easy to cause imbalance of neutral point voltage and distortion of terminal voltage between bridge legs when starting up due to not selecting redundant small vector. To this end, a new twelve-sectors-based SVPWM (TS-SVPWM) method is presented to solve this problem. It is obvious that the content and method that are studied in this paper will promote high reliability and perfect performance of EVs bidirectional charger, and help to develop new energy and smart grid. 


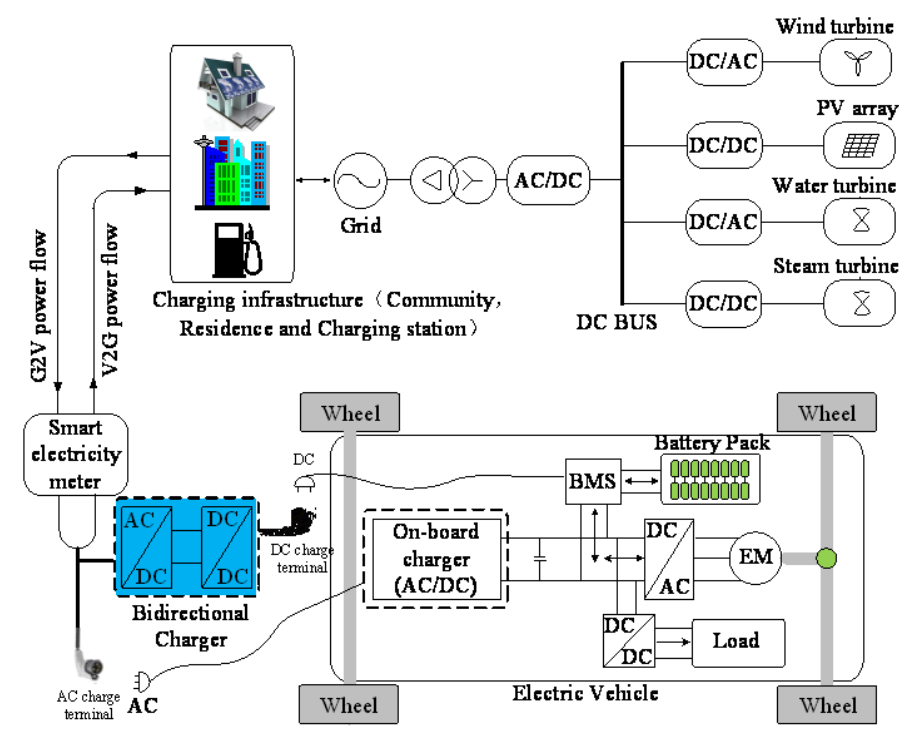

Fig.1: V2G technology applied to smart grid

\section{OPERATion Principle of Bidirectional Converter}

The main circuit topology of bidirectional EVs battery charger is composed of two-stage converter, the front-end converter is T-type NPC three phase TL AC/DC converter, and the second stage converter is series-parallel DAB converter, as is shown in Fig.2.

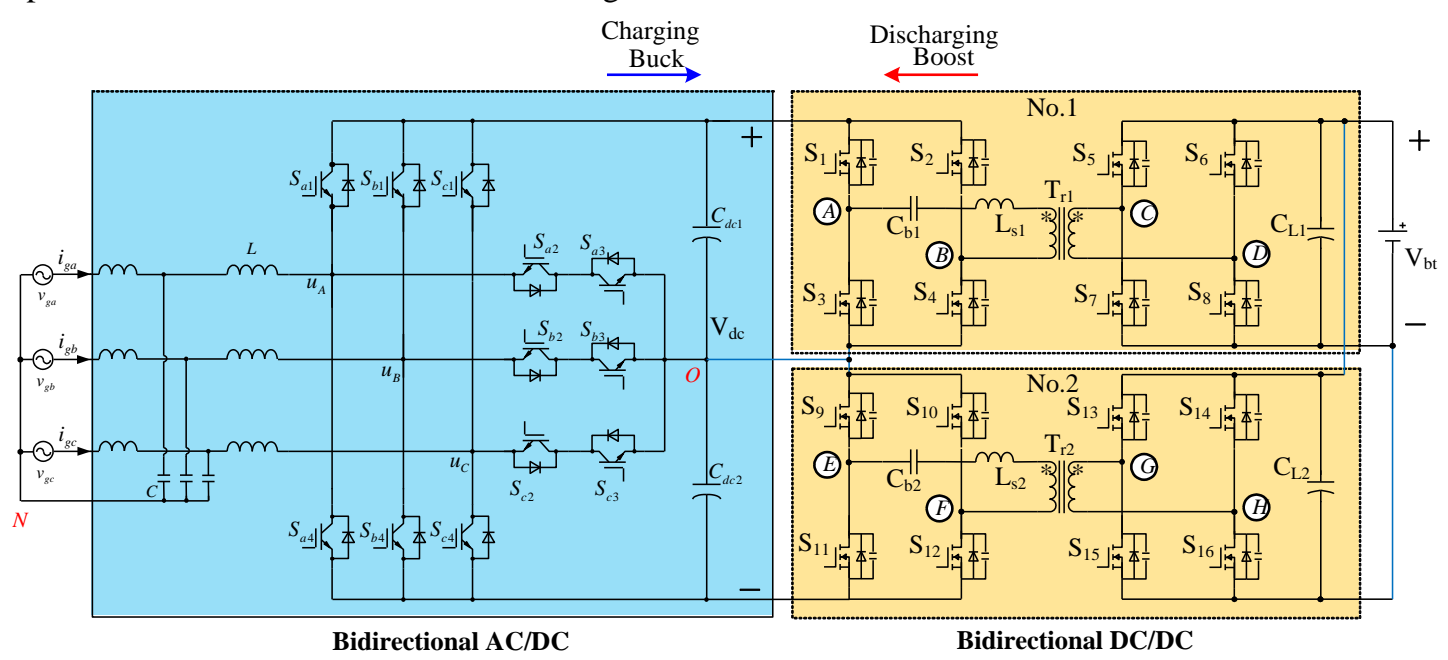

Fig. 2 Main circuit of isolated bidirectional charger

\section{A.Modulation Method of T-type Three-Phase TL Converter}

The sector classification figure of new twelve-sectors-based SVPWM algorithm is presented here, as shown in Fig.3, each of big sectors is divided into two small sectors compared to conventional SVPWM. So twelve sectors are gotten and followed by S1, S2, ...up to S12.

Take the small sectors in big sector I for example, if the reference vector meets $V_{\beta} \leq \sqrt{3} V_{\alpha} / 3$, the reference vector should locate in the small vector S1, or in S2. Discriminant analysis is similar to other small sector.

Take the small sectors of S1 and S2 for example, the operating sequences of switches are analyzed. As shown in the Fig.4, five-section-modulation method is adopted because the selectable vectors are rare. When the reference vector locates in the small sector $\mathrm{S} 1$, the operating sequence of vectors should be according to $\mathrm{V}_{0} \rightarrow \mathrm{V}_{7} \rightarrow \mathrm{V}_{13}$. When the reference vector locates in the small sector $\mathrm{S} 2$, the operating sequence of vectors should be according to $\mathrm{V}_{0} \rightarrow \mathrm{V}_{7} \rightarrow \mathrm{V}_{14}$. 


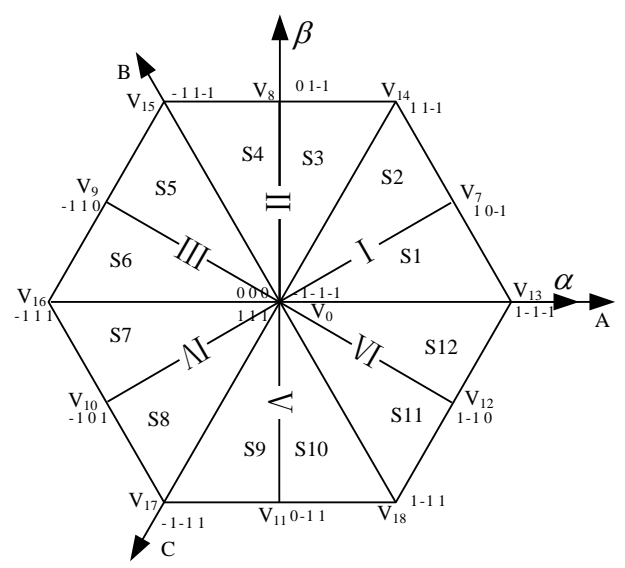

Fig.3: Twelve-sectors-based SVPWM

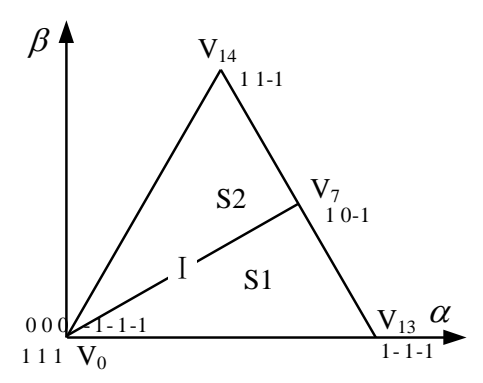

Fig.4: Sector I
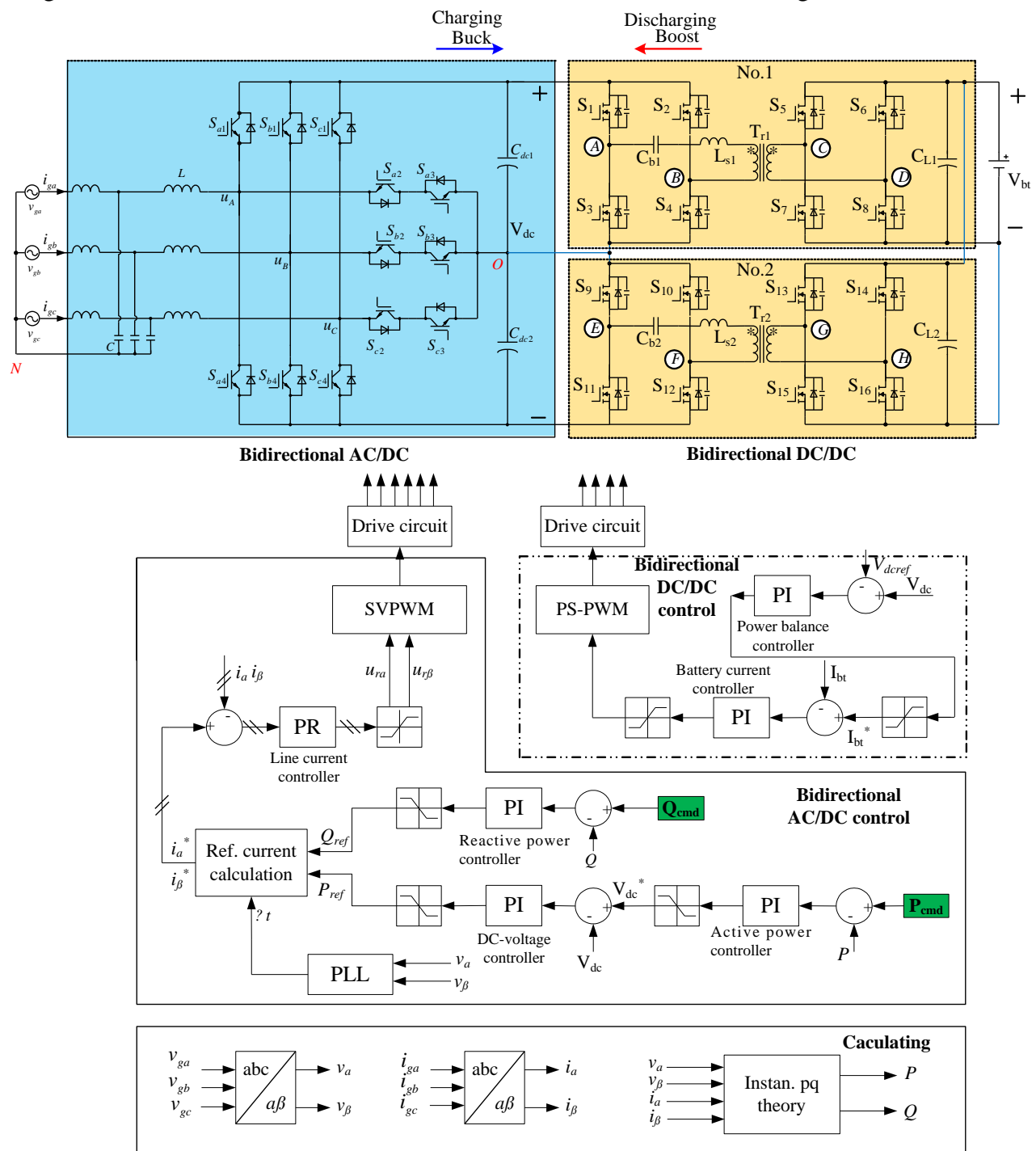

Fig.5: Direct power control strategy

\section{B. Series-Parallel Dual Active Bridge Converter}

The post stage circuit contains two DAB converters that called No.1 and No.2 DAB respectively as shown in the Fig.2, and the two input terminals of No.1 and No.2 DAB connect in series on the high-voltage side while the two output terminals are in parallel connection on the low-voltage side (Battery side). Since the DC-link voltage is up to $750 \mathrm{~V}$, the lower-voltage rated switch can be easily 
chosen due to SPDAB construction. This help to improve efficiency and reliability.

Energy flows from DC link to Battery when the phase of $\mathrm{V}_{\mathrm{AB}}$ leading that of $\mathrm{V}_{\mathrm{CD}}$, and the converter operates in Buck mode for charging battery; energy flows from Battery to DC link when the phase of $\mathrm{V}_{\mathrm{AB}}$ lagging that of $\mathrm{V}_{\mathrm{CD}}$, and the converter operates in Boost mode for discharging battery.

\section{Control Strategy BaSed on Direct PoWer}

The control strategy based on direct power is drawn in the Fig.5, and the two power references are from the power dispatching of grid. The electric quantities need to be sensed are as follows in Fig.5: grid ccurrent $i_{\mathrm{gi}}$ $(\mathrm{i}=\mathrm{a}, \mathrm{b}, \mathrm{c})$, grid voltage $v_{\mathrm{gi}}(\mathrm{i}=\mathrm{a}, \mathrm{b}, \mathrm{c})$, DC-link voltage $\mathrm{V}_{\mathrm{dc}}$ and battery current $\mathrm{I}_{\mathrm{bt}}$. Among these controller, power balance controller is also key except of important current controllers, and it is whether the performance of power balance controller is perfect or not that will have direct affect on control stability of two stage converters and power quality in both $\mathrm{AC}$ and DC sides. Coordinate transformations of the sensed grid voltages and grid currents are made to implement decoupled control of $\mathrm{P} / \mathrm{Q}$, and the real-time active power and reactive power calculated by the instantaneous active and reactive power theory, together with the power references from grid, construct negative feedback control to implement direct power control.

\section{EXPERIMENTAL RESULTS}

A $5 \mathrm{~kW}$ experimental prototype based two-stage main circuits as in Fig. 2 has been developed to verify the feasibilities, and main parameters are as follows: rated grid voltage is $220 \mathrm{~V} / 50 \mathrm{~Hz}$, inductance on $\mathrm{AC}$ side $\mathrm{L}=2.6 \mathrm{mH}$, the rated voltage of Lithium battery pack is $384 \mathrm{~V}, \mathrm{Ls} 1=\mathrm{Ls} 2=58 \mu \mathrm{H}, \mathrm{Cb} 1=\mathrm{Cb} 2=2 \mu \mathrm{F}$; Switches used in front-end converter choose IRG4PH40UDPBF from IXYS, and switching frequency is $15 \mathrm{kHz}$; Switches used in DAB converter choose IXFR36N60P from IXYS, and switching frequency is 40kHz. DSP TMS320F28335 from TI serves as main control chip to implement full digital control as in Fig.5.

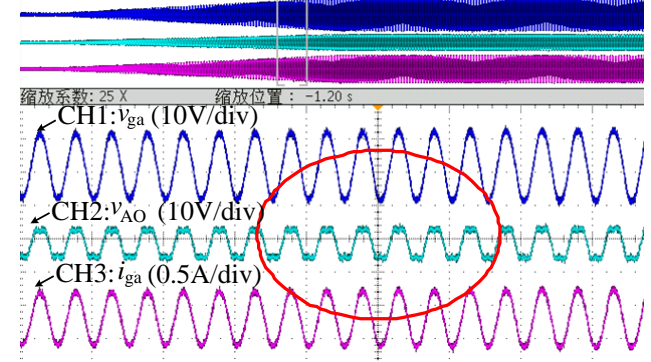

(a) Starting up waveforms

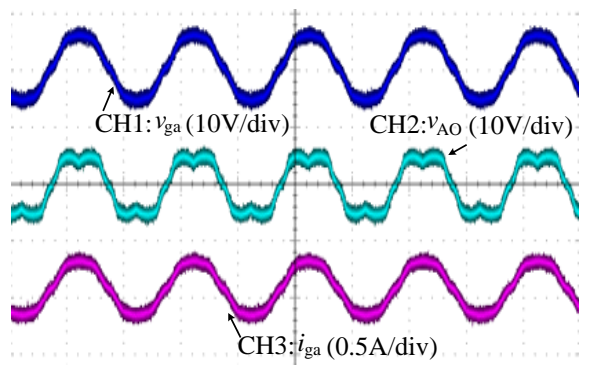

(b) Zoom waveforms (expanding waveforms)

Fig.6: Starting up waveforms of front-end AC/DC converter base on TS-SVPWM

The starting up waveforms of front-end T-type AC/DC converter is as in Fig.6, and the expanding waveforms in Fig.6(b) are corresponding to the waveforms cycled in Fig.6(a). It is seen that the terminal voltage waveform $v_{\mathrm{AO}}$ of bridge leg is perfect and without distortion due to that TS-SVPWM algorithm is adopted, of course, it is feasible.

As shown in the Fig.7 the drive signal $v_{\mathrm{gs} 1}$ on the high-voltage leads drive signal $v_{\mathrm{gs} 5}$ on the low-voltage side by phase, the charger operates in charging mode. As shown in the Fig.8 drain-source voltages of $S_{1}$ and $\mathrm{S}_{5}$ have been reduced to zero before these both switches switch on, as a consequence, it is seen that ZVS of switches can be achieved.

When the power references $\mathrm{P}_{\mathrm{cmd}}$ and $\mathrm{Q}_{\mathrm{cmd}}$, receiving from grid power dispatching, are $2160 \mathrm{~W}$ and \pm 1050Var respectively, the displacement factor is around \pm 0.9 . It is seen from Fig.9(a) that the inductive reactive compensation with $-1050 \mathrm{~V}$ ar and power flowing into grid is implemented due to direct power control strategy; and it is shown from Fig.9(b) that the capacitive reactive compensation with 1050Var and power flowing into grid is implemented due to direct power control strategy. In these two situations, bidirectional charger all operates in discharging mode. 


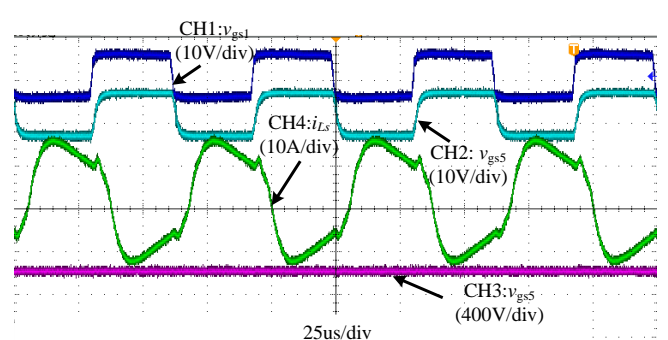

Fig.7: Operating waveforms when DAB converter charging battery

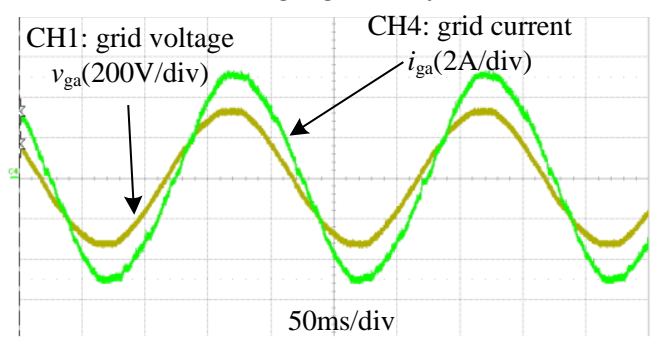

(a) $\lambda=-0.9, P_{\text {cmd }}=2160 \mathrm{~W}, Q_{\mathrm{cmd}}=-1050 \mathrm{Var}$

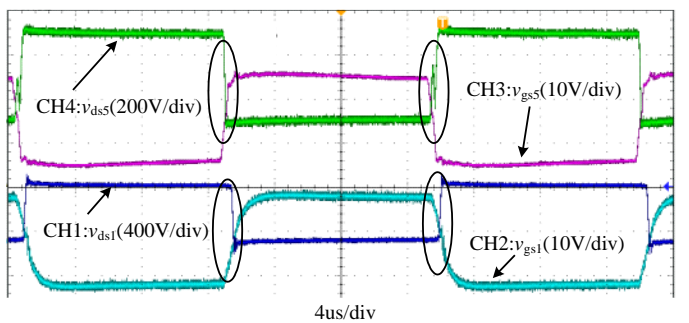

Fig.8: ZVS waveforms of DAB

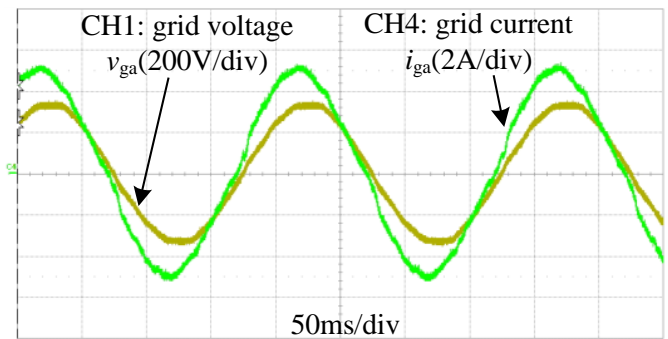

(b) $\lambda=0.9, \mathrm{P}_{\mathrm{cmd}}=2160 \mathrm{~W}, \mathrm{Q}_{\mathrm{cmd}}=1050 \mathrm{Var}$

Fig.9: Operating waveforms when charger supplying active power and reactive power with grid

\section{CONCLUSION}

The bidirectional charger studied in this paper consists of two stage converter. First, the T-type NPC three phase TL AC/DC converter is served as front-end circuits to interface local grid or micro grid. Second, the series-parallel DAB is configured as the post-stage circuit to implement electrical isolation between grid and battery. this bidirectional charger has high power density and safety. The terminal voltage waveform of bridge leg is not distorted when starting up due to the proposed TS-SVPWM method, and the switch is easy to be selected because of series-parallel structure in post-stage circuit, which help to improve cost performance. Most of important, the direct power based control strategy is easy to be implemented by digital design and receive power dispatching. the presented bidirectional charger can promote V2G application and development of smart grid.

\section{REFERENCES}

[1] Ming-Shi Huang,Po-Yi Yeh, Jia-Rong Huang, Chang-Hung Liao, "Novel bi-directional AC-DC converter for electrical vehicle battery testing," 37th Annual Conference on IEEE Industrial Electronics Society, Vol.7, No.11, pp.1480-1485, 2011.

[2] M. Yilmaz and P. T. Krein, "Review of battery charger topologies, charging power levels and infrastructure for plug-in electric and hybrid vehicles," IEEE Transaction on Power Electronics, vol. 28, pp. 2151-2169, May 2013.

[3] Mohammed Qasim, Parag Kanjiya, Vinod Khadkikar, "Artificial-neural-network-based phase-locking scheme for active power filters," IEEE Transactions on Industrial Electronics, Vol.61, No.8, 3857-3866, 2014.

[4] Qian Liu, Li Peng, Yong Kang, Shiying Tang, Deliang Wu, and Yu Qi, " A novel design and optimization method of an LCL filter for a shunt active power filter," IEEE Transactions on Industrial Electronics, Vol.61, No.8, pp.4001-4010, 2014.

[5] Huang Jun, Wang Yue and Gao Yuan, "on soft switching characteristics of dual active bridge converters applied in energy storage systems,"Electrical Drives,Vol.1, No.12, pp.74-78, 2012.

[6] Jorge L. Duarte, Marcel Hendrix, Marcelo Godoy Simões, "Three-port bidirectional converter for hybrid fuel cell systems," IEEE Transactions on Power Electronics, Vol.22, No.2, pp.40-47, 2007.

[7] R. Wang, F. Wang, D. Boroyevich, R. Burgos, R. Lai, P. Ning, and K. Rają, hhekara, "A high power density single-phase PWM rectifier with active ripple energy storage," IEEE Transaction on Power Electronics, vol. 26, no. 5, pp. 1430-1433, May 2011.

[8] Li Wen, "Research on transformerless three-phase three-level grid-connected inverters," Master's thesis, Yangzhou University, Yangzhou, Apr. 2015. 